\title{
Comparison of the effectiveness of bupropion versus buspirone in the treatment of hypoactive sexual desire in women, a randomized clinical trial
}

\begin{abstract}
Summary
Aim: To compare the effectiveness of bupropion versus buspirone in the treatment of hypoactive sexual desire in women.

Material and methods: Randomized clinical trial with 87 women diagnosed with hypoactive sexual desire, divided in 2 groups. Group A (average age: $47,46 \pm 4,83$ years) was treated with bupropion (150 mg daily) and Group B (average age: 45,78 $\pm 5,43$ years) with buspirone ( $20 \mathrm{mg}$ daily), during 6 months. Hypoactive sexual desire was evaluated using the questionnaire "Decreased Sexual Desire Screener".
\end{abstract}

Results: Women with hypoactive sexual desire at the beginning of the therapy didn't have a statistically significant increase in their sexual desire at the end of the study. In the analysis, there weren't detected statistically significant differences between the groups. Throughout the research, the continuation of the decrease in the sexual desire was statistically significant in both groups.

Conclusion: the treatment with bupropion and buspirone of the hypoactive sexual desire in women, it doesn't represent an effective therapeutics.

Keywords: coitus, feminine, women, sexuality, pharmacological treatment, hypoactive sexual desire, analysis, psychological, mental and social wellbeing, illness

\author{
Volume 2 Issue 5 - 2018
}

\author{
Franklin José Espitia De La Hoz,' Hoover \\ Orozco Gallego, ${ }^{2}$ Hugo Armando Gallego \\ Becerra $^{3}$ \\ 'Ginecología y Obstetricia, Universidad Militar Nueva Granada, \\ Colombia \\ ${ }^{2}$ Degree in Physics and Mathematics, Technological University of \\ Pereira-UTP, Colombia \\ ${ }^{3}$ Bachelor in Physics and Mathematics, Technological University \\ of Pereira-UTP, Dean of the basic sciences, Colombia
}
Correspondence: Franklin José Espitia De La Hoz, Gynecology and Obstetrics, New Granada Military University, Clinical Sexology, Master in Sexology: Education and sexual counseling, Service of Gynecology and Fetal Maternal Medicine, La Sagrada Familia Clinic, Armenia, Quindío, Colombia, Tel 7459490,3127436696,Email espitiafranclin@hotmail.com

Received: September 13,2018 | Published: October 30, 2018
Abbreviations: HSDD, hypoactive sexual desire disorder; DSDS, decreased sexual desire screener; PDE-5, phosphodiesterase-5

\section{Introduction}

Sexual health is defined by the World Health Organization as a state of physical, psychological, mental and social wellbeing in relation with sexuality; therefore, it's not only the absence of illness. ${ }^{1}$ The definition is based on historical publications of sexual health researchers during the last century. Once normal is defined, what is "abnormal" can be recognized, defined and finally be treated. The decrease of the sexual desire is the most widely known and reported sexual complain ${ }^{2-4}$ in both sexes; however, despite being the most prevailing sexual disorder in women (it varies between $8 \%$ and $34.9 \%$ ), of all ages, it's also one of the most difficult to approach. ${ }^{5-7}$ The diagnostics of the hypoactive sexual desire disorder (HSDD) in women, begins with the preparation of the records of a complete medical, psychosocial and sexual history; it must be given a full physical examination, including an exhaustive gynecological exploration to determine to determine if the cause has a biological, psychological, sociocultural origin or its partner related or any combination of the above. ${ }^{5-7}$ Although there are many self-evaluation questionnaires, made with the purpose of determining the clinical evaluation of the feminine hypoactive sexual desire disorder, the Decreased Sexual Desire Screener (DSDS) is a validated instrument used by physicians, and which was used in this research. ${ }^{8,9}$ The pharmacological treatments for HSDD include homeopathic and hormonal products, phosphodiesterase-5 (PDE-5) inhibitors, psychotropic drugs, etc. ${ }^{10-12}$ This variety of therapeutic options is because of the poor understanding that we still have over the biology of desire. Bupropion is a reuptake inhibitor of norepinephrine and dopamine approved by the FDA for depression treatment. It's been studied because of the effect over the sexual functioning and
HSDD with results that show an improved sexual desire in women with depression, as well as an improvement of desire in women with HSDD induced by reuptake inhibitors of serotonin. ${ }^{13-15}$ In short, it has been credited with a positive effect on the increase of sexual desire. Buspirone is a non-benzodiazepine anxiolytic whose mechanism of action is not clearly established. It acts as a partial antagonist or with a mixed antagonism/agonism of the 5-HT1A receptors to which it owes its anxiolytic effects; it also has a moderate affinity towards type-2 dopaminergic receptors (DA2), showing antagonist and dopaminergic agonist effects. ${ }^{17}$ It's been suggested that improves the sexual functioning by increasing the libido. ${ }^{18}$ The aim of this study is to compare the effectiveness of bupropion in the treatment of hypoactive sexual desire in women versus buspirone.

\section{Material and methods}

A randomized, double blind, prospective clinical trial type of research was designed, with a total duration of 6 months. Women diagnosed with hypoactive sexual desire disorder (HSDD) were part of it; they were recruited during the consultation with the author who gave their written consent, signing the informed agreement to participate in the research, after knowing about its objectives. The criteria of inclusion were women diagnosed by the sexologist with hypoactive sexual desire who met the criteria of the Decreased Sexual Desire Screener (DSDS), with symptomatology that interfered with their sexual activities at the time of the beginning of the study, and who were eligible to receive treatment with bupropion or buspirone. The DSDS is a validated instrument to be used by physicians to help in the diagnosis of HSDD. ${ }^{8,9}$ Among other advantages, it's brief, it can be used both in premenopausal and postmenopausal women, it's useful for physicians who are not experts in sexual disorders. ${ }^{8}$ It's used in a self-administered way and it consists of 5 questions (Table 
1). Its diagnostic accuracy is $85.2 \%$ (sensitivity and specificity of 83.6-95.6\% and $87.8 \%$, respectively). ${ }^{19,20}$ The first four questions evaluate if the woman has lost sexual desire and if she feels affected or bothered by it, the fifth compound question, helps the doctor to perform a differential diagnosis. If an answer is affirmative, in any of the sections of the fifth question, it doesn't exclude the possibility of a diagnostic test of hypoactive sexual desire disorder and it constitutes an alarm for the doctor to discuss the issue with her.

Table I Decreased Sexual Desire Screener (DSDS): brief diagnostic assessment for generalized, acquired hypoactive sexual desire disorder

$\begin{array}{ll}\text { I. In the past, was your level of sexual desire or interest good and satisfying to you? } & \text { Yes No } \\ \text { 2. Has there been a decrease in your level of sexual desire or interest? } & \text { Yes No } \\ \text { 3.Are you bothered by your decreased level of sexual desire or interest? } & \text { Yes No } \\ \text { 4.Would you like your level of sexual desire or interest to increase? } & \text { Yes No }\end{array}$

5. Please circle all the factors that you feel may be contributing to your current decrease in sexual desire or interest:

A) An operation, depression, injuries, or other medical condition.

Yes No

B) Medications, drugs, or alcohol you are currently taking.

Yes No

C) Pregnancy, recent childbirth, menopausal symptoms.

Yes No

D) Other sexual issues (pain, decreased arousal or orgasm)

Yes No

E) Your partner's sexual problems.

Yes No

F) Dissatisfaction with your relationship or partner.

Yes No

G) Stress or fatigue

Yes No

Doctor:

If the patient answers "NO" to any of the first 4 questions, then she doesn't qualifies for the diagnosis of acquired HSDD.

If the patient answers "YES" to all of the first 4 questions and her review confirms "NO" to all the factors in question 5 , then she may qualify for the diagnosis of acquired HSDD.

If the patient answers "YES" to all of the first 4 questions and "YES" to any of the factors in question 5, then you must decide whether the answers in the fifth question indicate a primary diagnosis different from that of acquired HSDD. Concurrent illnesses associated with arousal or orgasm should not exclude the realization of a simultaneous diagnosis of HSDD

Based on the above, does the woman suffer acquired hypoactive sexual desire disorder?

Yes No

Pregnant and epileptic women and those with some type of psychiatric illness, with a history of drug addiction and/or alcoholism, who had several diagnoses of sexual disorders, who received another type of treatment due to hypoactive sexual desire at the beginning of the study or that they had received it in the previous 6 months, were excluded and those that did not consent to participate in the research. Sampling was done by convenience of the women who came to the consultation with the main author of the research; those who met the criteria and accepted to participate in the study were included in a consecutive way. The women were divided into two groups through a system of simple randomization of computer generated random numbers: one group (A) received $150 \mathrm{mg}$ of prolonged-release bupropion and the other group (B) received $20 \mathrm{mg}$ of buspirone. Before the experiment, each envelope with the tablets was masked, so that neither the doctor nor the patient knew what medication was receiving, thus the experiment was blinded. Each patient was given an envelope containing the appropriate number of tablets for the first seven days of treatment; after the first medical check-up and confirming that they had consumed the tablets, they were given a second envelope to complete the three-week treatment, and so on successively in each control. The tablets were given free of charge. They were instructed to return any unused medication, as well as to report the presence of adverse or undesirable effects. The following outcomes were measured: the time since diagnosis (in years), the increase in sexual desire during the treatment, sexual satisfaction, coital frequency during the treatment and at the end of it and presence of adverse or undesirable effects.

It was determined that the treatment was effective when the increase in sexual desire was rated by the patient as above $51 \%$ (51$74 \%$ was considered a slight increase and $>75 \%$ a significant increase). The increase in desire was rated with a verbal scale of 4 points $(0=$ no relief, $1=$ little relief, $2=$ moderate relief, $3=$ complete relief $)$. If after six months of treatment (end of the study), the percentage of improvement was equal to or less than $50 \%$, it was deemed as a therapeutic failure. Five follow-ups were performed, at the beginning of the trial, after one week, after three weeks, after three months and at the end of the study. The initial evaluation was prior to randomization, and it recorded the socio-demographic data, time of evolution of the disorder from diagnosis, sexual satisfaction and coital frequency. During the follow-up and at the end of the study, the data obtained was again recorded, as well as the effectiveness of the increase in sexual desire. The records were made in the same environment, at the same time, with the same measuring instruments and by the same examiner as in the initial evaluation. This study follows the principles of the Declaration of Helsinki. The confidentiality of the information and the privacy of the participating women were guaranteed.

\section{Data analysis}

The computation of the categorical variables was reported in absolute numbers and percentages. The differences in baseline and post-intervention characteristics were estimated through hypothesis testing. In the qualitative variables, the $\chi 2$ test and Fisher's exact test were used, as appropriate. The baseline characteristics of both groups were presented in a table using measures of frequency and proportions for qualitative variables, and measures of central tendency and dispersion for variables of a quantitative nature. The analysis was done with the help of the SPSS software version 20. 


\section{Results}

87 women were part of this study, they were diagnosed with hypoactive sexual desire and were $47.46 \pm 4.83$ years old in average in group A and $45.78 \pm 5.43$ years old in average in group B. Figure 1 shows the flowchart of the process of the participants throughout the study. At the end of it, there was an exclusion of five women (three in group A and two in group B), so the final analysis was for 42 women in group A and 40 women in group B. The socio-demographic characteristics of the sample are described in Table 2. It stands out that $64.56 \%$ of the women lived in a free union, $69.51 \%$ were housewives, and $65.85 \%$ had 1 to 2 children. The predominant racial group was Hispanic $(45.12 \%)$. No statistically significant differences were found between the two study groups in regards to socio-demographic variables (age, marital status, occupation, weight, etc.) $(\mathrm{p}>0.05)$. The average time of evolution of the sexual disorder for the total sample varied between 3 and 12 years, so the average for group A was $7.83 \pm 1.59$ years and $6.78 \pm 1.32$ years for group B. It wasn't found a statistically significant difference in their sexual satisfaction (7.14\% for group A and 10\% for group B, $\mathrm{p}=0.816$ ) (Table 2). Most of the women declared a low frequency of coital sexual encounters after one week (less than 1), without any statistically significant difference between the two groups $(\mathrm{p}=0.153) .28 .57 \%(\mathrm{n}=12)$ versus the $27.5 \%(n=11)$ of the women in the bupropion and buspirone group respectively, increased the sexual frequency (1-2 times a week) in the first 3 weeks after the beginning of the study. $(\mathrm{p}>0.05)$. Similarly, $71.42 \%(\mathrm{n}=30)$ versus $72.5 \%(\mathrm{n}=29)$ of the patients in the bupropion and buspirone group respectively, kept a low sexual frequency during the first three weeks $(p>0.05)$. In the following intervals, up to six months after the research, no significant differences were observed between the groups. The baseline evaluation of the coital frequency was similar in both groups $(\mathrm{p}=0.27)$, this similarity continued in the test performed at 3 months $(p=0.33)$ and at 6 months $(p=0.15)$. In the tests (baseline, after a week and after 6 months) there was no statistically significant difference $(\mathrm{p}>0.05)$.

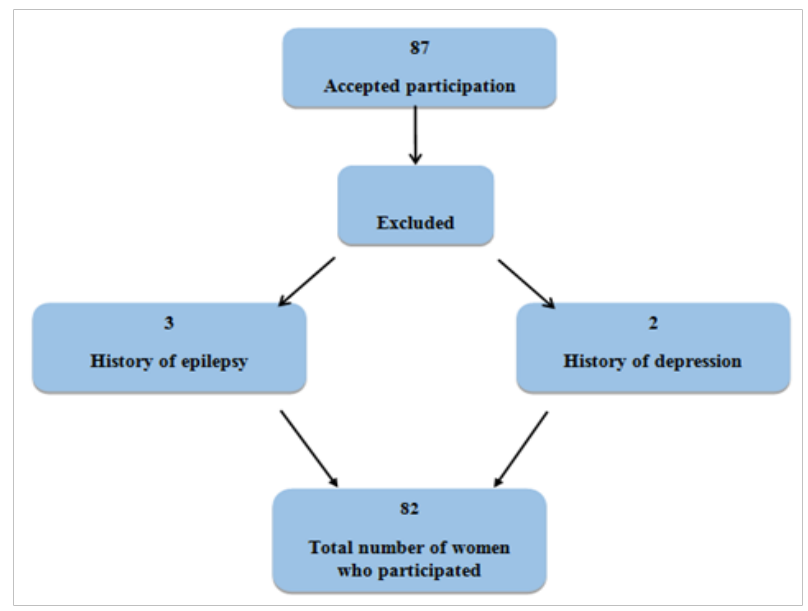

Figure I Flowchart of women with HSDD who participated in the study.

No significant difference was found in the improvement of sexual desire, after six months of treatment, in both groups $(\mathrm{p}=0.708)$, nor in the frequency of sexual encounters $(p=0.243)$. The implementation of the DSDS at 3 and 6 months showed that there were no changes in either the frequency of coital or sexual satisfaction, compared to the start of the study; and there were no statistically significant differences in the comparison between the evaluations at 3 and 6 months, both for the coital frequency $(p=0.648)$ and for sexual satisfaction $(p=0.696)$.
Adverse effects such as agitation, insomnia, nausea, drowsiness, tachycardia, tremors, and some gastrointestinal symptoms were reported. The incidence of tachycardia and tremors was $9.52 \%$ (four patients); in the bupropion group, the incidence of nausea and drowsiness was $10.5 \%$ (six patients) for the buspirone group, with no statistically significant differences between them $(p=1)$. At the end of the study, $85.71 \%$ from group A and $82.5 \%$ from group B claim that their sexual desire has not improved enough. $92.85 \%$ from group A and $90 \%$ from group B report that their sexual frequency remains the same as when they started the trial. $90.47 \%$ of the women from group $\mathrm{A}$ and $87.5 \%$ from group $\mathrm{B}$, consider the treatment useless. It was noted that satisfaction with both therapeutic guidelines was low (9.52\% for group A and $12.5 \%$ for group B), without differences between the two groups.

Table 2 Socio-demographic characteristics of women with Hypoactive Sexual Desire Disorder

\begin{tabular}{llll}
\hline & Group A $(\mathbf{n}=\mathbf{4 2})$ & Group B $(\mathbf{n}=\mathbf{4 0})$ & $\mathbf{p}$ \\
\hline Age & $47,46 \pm 4,83$ & $45,78 \pm 5,43$ & 0.549 \\
Weight $(\mathrm{Kg})$ & $61.2 \pm 8.73$ & $60.6 \pm 8.46$ & 0.708 \\
BMI $\left(\mathrm{Kg} / \mathrm{m}^{2}\right)$ & $25.2 \pm 2.4$ & $24.6 \pm 2.7$ & 0.609 \\
Number of children & $\mathrm{I}, 59 \pm \mathrm{I}, \mathrm{I4}$ & $\mathrm{I}, 47 \pm \mathrm{I}, 32$ & $0.41 \mathrm{I}$
\end{tabular}

\section{Education}

\begin{tabular}{|c|c|c|c|}
\hline Primary school \% & 12 & 13 & 0.513 \\
\hline Secondary school \% & 21 & 19 & 0.429 \\
\hline University \% & 9 & 8 & 0.636 \\
\hline \multicolumn{4}{|l|}{ Marital Status } \\
\hline Married \% & 6 & 7 & 0.576 \\
\hline Free union \% & 27 & 24 & 0.903 \\
\hline Separated \% & 6 & 5 & 0.369 \\
\hline Divorced \% & 3 & 4 & 0.423 \\
\hline \multicolumn{4}{|l|}{ Origin } \\
\hline Rural \% & 15 & 14 & 0.408 \\
\hline Urban \% & 27 & 26 & 0.729 \\
\hline \multicolumn{4}{|l|}{ Occupation } \\
\hline Housewife \% & 29 & 28 & 0.417 \\
\hline Employee & 13 & 12 & 0.144 \\
\hline \multicolumn{4}{|l|}{ Race } \\
\hline Afro-Colombian & 15 & 14 & 0.717 \\
\hline Hispanic & 18 & 19 & 0.792 \\
\hline Native & 9 & 7 & 0.597 \\
\hline $\begin{array}{l}\text { Time since diagnosis } \\
\text { (years) }\end{array}$ & $7.83 \pm 1.59$ & $6.78 \pm 1.32$ & 0.387 \\
\hline Sexual satisfaction & $7.14 \%$ & $10 \%$ & 0.816 \\
\hline Coital frequency & $<$ I week & <I week & 0.153 \\
\hline
\end{tabular}

\section{Discussion}

In this research we found that bupropion and buspirone are similar in the lack of effectiveness for the treatment of HSDD in women. These results reassert, when we talk about hypoactive sexual desire, and despite of more than three decades from its initial definition, as well as multiple changes trying to improve the accuracy of the 
diagnosis, that we still need to set much more effective therapeutic paradigms. Unlike other studies, ${ }^{10-22}$ the bupropion didn't show an increase either in the sexual desire nor the frequency, let alone any subjective range of sexual satisfaction. The preparation of prolongedrelease bupropion has been described as being able to improve sexual desire and orgasmic disorder in women with hypoactive sexual desire, even in the absence of depression; ${ }^{23}$ however, the findings of this study indicate that bupropion is ineffective for the treatment of HSDD. The changes reported by the women who received it in this research showed a sexual functioning that did not favor it, inconsistent with what was reported by other authors. ${ }^{10-24}$ The results of this research are in agreement with other publications, where the use of buspirone is ineffective in the treatment of HSDD in women, since it did not show statistically significant differences with a placebo. ${ }^{25,26}$ Although we expected a better response rate, in both groups, at the registered dose; after three months of follow-up, an increase in the dose was not considered because the reviewed studies showed no benefits from increasing it. ${ }^{21-26}$ In the reviewed literature, we didn't find previous studies that compared the effectiveness of bupropion and buspirone in the treatment of women's HSDD. However, let's consider, after this research, despite the small sample, that new studies will complete other results or show beneficial findings in relation to HSDD.

One explanation for the poor response, with the drugs studied, is that their lack of efficacy is due to the fact that they are not useful in patients without depression. You can say that bupropion and buspirone don't have a better behavior than other drugs in the treatment of HSDD in women, however, it must be clear that, in the case of female sexuality, the purpose is not only to achieve the increase of sexual desire, sexual frequency and sexual satisfaction, but the recovery of their sexual environment at all costs. After reviewing the different adverse effects of bupropion and buspirone, we observed that they seemed to be less frequent than those reported in other studies. ${ }^{23-26}$ To the date of completion of this research, it's known that clinical trials are being conducted to evaluate different medications that may benefit women with hypoactive sexual desire; which we hope will be more promising than those studied here. Sexual disorders in women, are strongly associated with feelings of physical and emotional dissatisfaction, as well as low feelings of happiness; ; 5 which makes hypoactive sexual desire to have a significant impact on the quality of life; Therefore, from the results of this study we suggest to continue investigating new therapeutic options that allow us to have the right alternative when choosing a drug. Among the limitations of the study, you can find the small number of the sample, the short follow-up time and the subjective way of measuring the variables (coital frequency, sexual satisfaction and increased sexual desire). Although sexual satisfaction was low in both groups, it's possible that women with depression have a better response. The strong point being the first research carried out in Colombia, with two non-hormonal therapies, handling HSDD in non-depressed women. We extend the invitation about the urgent need for new research that evaluates other therapeutic alternatives in handling of women's HSDD.

\section{Conclusion}

The use of these drugs is a poor pharmacological tool, in terms of utility and effectiveness in the treatment of hypoactive sexual desire in women, given that it provides an inefficient increase in sexual desire, with a very low satisfaction rate.

\section{Thanks}

To M.D. Francisco Cabello Santamaría, great teacher and researcher, for sowing in me the curiosity of the approach and handling of hypoactive sexual desire in women; without his motivation this research wouldn't have been possible.

\section{Financing}

This study was financed thanks to the main author's own resources.

\section{Acknowledgements}

None.

\section{Conflict of interest}

The authors declare no conflict of interest.

\section{References}

1. WHO. World Health Organization. Education and Treatment in Human Sexuality: The training of health professionals. Technical Report Series 572. Geneva: WHO; 1975 .

2. Knoepp LR, Shippey SH, Chen CCG, et al. Sexual complaints, pelvic floor symptoms, and sexual distress in women over forty. $J$ Sex Med. 2010;7(11):3675-82.

3. Laumann EO, Paik A, Rosen RC. Sexual dysfunction in the United States: prevalence and predictors. JAMA. 1999;281(6):537-44.

4. American College of Obstetricians and Gynecologists Committee on Practice Bulletins-Gynecology ACOG practice bulletin No 119: female sexual dysfunction. Obstet Gynecol. 2011;117(4):996-1007.

5. Espitia De La Hoz, FJ. Prevalence of sexual dysfunction in women of the Coffee Region. Rev cienc biomed. 2016;7(1):25-33.

6. Espitia-De La Hoz FJ. Evaluation of the prevalence of sexual dysfunction in female physicians, from the Colombian Coffee Region, in the climacteric stage. Arch Med (Manizales). 2017;17(1):70-77.

7. Shifren JL, Monz BU, Russo PA, et al. Sexual problems and distress in United States women: prevalence and correlates. Obstet Gynecol. 2008;112(5):970-978.

8. Clayton AH, Goldfischer ER, Goldstein I, et al. Validation of the Decreased Sexual Desire Screener (DSDS): a brief diagnostic instrument for generalized acquired female hypoactive sexual desire disorder (HSDD). J Sex Med. 2009;6(3):730-738.

9. Clayton AH, Goldfischer E, Goldstein I, et al. Validity of the Decreased Sexual Desire Screener for diagnosing hypoactive sexual desire disorder. J Sex Marital Ther. 2013;39(2):132-143.

10. Segraves RT, Croft H, Kavoussi R, et al. Bupropion sustained release (SR) for the treatment of hypoactive sexual desire disorder (HSDD) in nondepressed women. J Sex Marital Ther. 2001;27(3):303-316.

11. Nurnberg HG, Lauriello J, Hensley PL, et al. Sildenafil for iatrogenic serotonergic antidepressant medication-induced sexual dysfunction in 4 patients. J Clin Psychiatry. 1999;60(1):33-35.

12. Lobo RA, Rosen RC, Yang HM, et al. Comparative effects of oral esterified estrogens with and without methyltestosterone on endocrine profiles and dimensions of sexual function in postmenopausal women with hypoactive sexual desire. Fertil Steril. 2003;79(6):1341-1352.

13. Segraves RT, Croft H, Kavoussi R, et al. Bupropion sustained release (SR) for the treatment of hypoactive sexual desire disorder (HSDD) in nondepressed women. J Sex Marital Ther. 2001;27(3):303-316.

14. Clayton AH, McGarvey EL, Warnock JK. Bupropion as a treatment for SSRI-induced sexual dysfunction; Female Sexual Functioning Forum. 2000;26-29. 
15. Gitlin MJ. Effects of depression and antidepressants on sexual functioning Bull Menninger Clin. 1995;59(2):232-248.

16. Segraves RT, Clayton A, Croft H, et al. Bupropion sustained release for the treatment of hypoactive sexual desire disorder in premenopausal women. J Clin Psychopharmacol. 2004;24(3):339-342.

17. Apter JT, Allen LA. Buspirone: future directions. J Clin Psychopharmacol. 1999;19(1):86-93.

18. Michelson D. Female sexual dysfunction associated with antidepressant administration: a randomized, placebo-controlled study of pharmacologic intervention. Am J Psychiatry. 2000;157(2):239-243.

19. Goldfischer ER, Clayton AH, Goldstein I. Decreased Sexual Desire Screener (DSDS) for diagnosis of Hypoactive Sexual Desire Disorder in women. Obstet Gynecol. 2008;111(Suppl 1):109S.

20. Nappi RE, Dean J, Hebert A. Decreased Sexual Desire Screener (DSDS) for diagnosis of Hypoactive Sexual Desire Disorder (HSDD) in European women. J Sex Med. 2009;6(Suppl 2):46.

21. Safarinejad M, Hosseini S, Asgari M, et al. A randomized, double-blind, placebo-controlled study of the efficacy and safety of bupropion for treating hypoactive sexual desire disorder in ovulating women. BJU Int. 2010;106(6):832-839.

22. Crenshaw TL, Goldberg JP, Stern WC. Pharmacologic modification of psychosexual dysfunction. $J$ Sex Marital Ther. 1987;13(4):239-253.

23. Modell JG, May RS, Katholi CR. Effect of bupropion-SR on orgasmic dysfunction in nondepressed subjects: a pilot study. $J$ Sex Marital Ther. 2000;26(3):231-240.

24. Stahl SM, Pradko JF, Haight BR, et al. A review of the neuropharmacology of bupropion, a dual norepinephrine and dopamine reuptake inhibitor. Prim Care Companion J Clin Psychiatry. 2004;6(4):159-66.

25. Landen M, Eriksson E, Agren H, et al. Effect of buspirone on sexual dysfunction in depressed patients treated with selective serotonin reuptake inhibitors. J Clin Psychopharmacol. 1999;19(3):268-271.

26. Poels S, Bloemers J, van Rooij K, et al. Two novel combined drug treatments for women with hypoactive sexual desire disorder. Pharmacol Biochem Behav. 2014;121:71-79. 